\title{
On a generalization of the risk model with Markovian claim arrivals
}

\author{
Eric C.K. Cheung *, David Landriault ${ }^{\dagger}$ and Andrei L. Badescu ${ }^{\ddagger}$
}

March 24, 2011

\begin{abstract}
The class of risk models with Markovian arrival process (MAP) (see e.g. Neuts (1979)) is generalized by allowing the waiting times between two successive events (which can be a change in the environmental state and/or a claim arrival) to have an arbitrary distribution. Using a probabilistic approach, we determine the solution for a class of Gerber-Shiu functions apart from some unknown constants when claim sizes have a mixed exponential distribution. Such constants are later determined using the more classic ruin-analytic approach. A numerical example is later considered to illustrate the tractability of the suggested methodology in the study of Gerber-Shiu functions.
\end{abstract}

\section{Introduction}

In the last two decades, there has been an accrued interest in ruin theory for surplus processes defined with an underlying Markovian process. For instance, the Markov-modulated risk model and the risk model with Markovian arrival process (MAP) have been extensively studied by various authors (see e.g. Ahn et al. (2007), Asmussen (1989), Badescu et al. (2005), Cheung and Landriault (2009), Li and Lu (2007) and references therein).

In the MAP risk model for instance, there exists a homogeneous continuous-time Markov chain (CTMC), say $J=\{J(t), t \geq 0\}$, with finite state space $E=\{1,2, \ldots, m\}$ which describes the evolution of an (unobservable) environmental process. Two types of transition may occur in a MAP risk model:

(1) transitions of the CTMC $\underline{J}$ from state $i$ to state $j(j \neq i)$ without a claim (type- 1 transitions); or

(2) transitions of the CTMC $\underline{J}$ from state $i$ to state $j$ (with possibly $i=j$ ) with an accompanying claim (type-2 transitions).

In what follows, we refer to either type of transition as a system change. Type-1 transitions are governed by the matrix $\mathbf{D}$, for which its $(i, j)$-th element $D_{i j}\left(D_{i j} \geq 0\right)$ corresponds to the instantaneous

\footnotetext{
*Department of Statistics and Actuarial Science, University of Hong Kong, Pokfulam, Hong Kong

${ }^{\dagger}$ Department of Statistics and Actuarial Science, University of Waterloo, 200 University Avenue West, Waterloo, Ontario, Canada

${ }^{\ddagger}$ Department of Statistics, University of Toronto, 100 St. George St., Toronto, Ontario, Canada
} 
rate of transition from state $i$ to state $j(j \neq i)$ in $E$ without an accompanying claim. Type- 2 transitions are governed by the matrix $\mathbf{T}$, for which its $(i, j)$-th element $T_{i j}\left(T_{i j} \geq 0\right)$ corresponds to the instantaneous rate of transition from state $i$ to state $j$ in $E$ with an accompanying claim. The diagonal elements of $\mathbf{D}$ are assumed to be negative such that the sum of the elements on each row of the matrix $\mathbf{D}+\mathbf{T}$ is zero. We remark that the Markov modulated risk model (see e.g. Asmussen (1989) and Li and Lu (2007)) and Albrecher and Boxma (2005)'s semi-Markovian risk model can be retrieved from the MAP risk model by respectively letting the matrices $\mathbf{T}$ and $\mathbf{D}$ to be diagonal.

From the definition of the MAP risk model, it is implicitly assumed that the waiting times between two successive system changes are all exponentially distributed. More precisely, given that the underlying state of the CTMC $\underline{J}$ at a given time is $i$, it is assumed that the time until the next system change is exponentially distributed with mean $-1 / D_{i i}$. Also, the probability that the system change is a transition of the CTMC $\underline{J}$ from state $i$ to state $j$ with (without) a claim is given by $-T_{i j} / D_{i i}\left(-D_{i j} / D_{i i}\right.$ for $\left.j \neq i\right)$.

In this paper, we propose to extend the class of MAP risk models by assuming an arbitrary distribution for the time between two successive system changes. Thus, our generalized version of the MAP risk model allows the selection of a heavy-tailed distribution (e.g. a Pareto or a lognormal distribution) as well as other distributions (e.g. a Weibull or a gamma distribution) for the time between two successive system changes.

With that in mind, we introduce the risk model of interest in this paper. Let $Z_{0}$ be the current (time 0 ) environmental state and $Z_{i}$ be the environmental state at the time of the $i$-th system change. We assume that the sequence $\left\{Z_{i}\right\}_{i=0}^{\infty}$ is a homogeneous and irreducible discrete-time Markov chain on a finite state space $E=\{1,2, \ldots, m\}$. The one-period transition probability of the Markov chain $\left\{Z_{i}\right\}_{i=0}^{\infty}$ is

$$
\mathbf{G}=\mathbf{P}+\mathbf{Q}
$$

where $\mathbf{P}=\left[p_{i j}\right]_{i, j=1}^{m}$ and $\mathbf{Q}=\left[q_{i j}\right]_{i, j=1}^{m}$ with $p_{i j}, q_{i j} \geq 0$ and $(\mathbf{P}+\mathbf{Q}) \mathbf{1}=\mathbf{1}$ (1 being a column vector of 1s of appropriate dimension). Note that the one-period transition probability $\mathbf{G}$ has been expressed as $\mathbf{P}+\mathbf{Q}$ given that, as in the MAP risk model, two types of system changes may occur: (1) a change in the environmental process without a claim; or (2) a change in the environmental process accompanied by a claim. The probabilities of those scenarios are respectively contained in the matrices $\mathbf{P}$ and $\mathbf{Q}$. Note that the diagonal elements of $\mathbf{P}$ are all zero due to the definition of a system change (i.e. $p_{i i}=0$ for $i=1,2, \ldots, m)$. Finally, let $\pi_{i}$ be the long-run proportion of time that the Markov chain $\left\{Z_{i}\right\}_{i=0}^{\infty}$ is in state $i$. Since $\left\{Z_{i}\right\}_{i=0}^{\infty}$ is assumed to be irreducible, the long-run proportions $\left\{\pi_{i}\right\}_{i=1}^{m}$ are known to be the unique solution of the system

$$
\begin{aligned}
\pi_{j} & =\sum_{i=1}^{m} \pi_{i}\left(p_{i j}+q_{i j}\right), \quad j=1,2, \ldots, m \\
\sum_{i=1}^{m} \pi_{i} & =1 .
\end{aligned}
$$

Let $T_{0}=0$ and $T_{i}$ be the time of the $i$-th system change $(i=1,2, \ldots)$. For convenience, we define the counting process of the system changes, namely $\underline{N}=\{N(t), t \geq 0\}$, by $N(t)=\sup \left\{i \in \mathbb{N}: T_{i} \leq t\right\}$. We also introduce a sequence $\left\{W_{i}\right\}_{i=1}^{\infty}$ where $W_{1}=T_{1}$ is the time of the first system change and $W_{i}=T_{i}-T_{i-1}$ $(i=2,3, \ldots)$ is the time elapsed between the $(i-1)$-th and the $i$-th system changes. We assume that 
$W_{i} \mid Z_{i-1}=j$ has density function $k_{j}$, cumulative distribution function (c.d.f.) $K_{j}$, Laplace transform $\widetilde{k}_{j}(s)=\int_{0}^{\infty} e^{-s t} k_{j}(t) d t$ and mean $\kappa_{j}$. Conditional on $\left\{Z_{i}\right\}_{i=0}^{\infty}$, the random variables (r.v.'s) $W_{1}, W_{2}, \ldots$ are all independent.

Let $X_{i}$ be the claim size accompanying the $i$-th system change. We assume that $X_{i}=0$ if the $i$-th system change does not involve a claim while $X_{i}$ has density $f_{j k}$, c.d.f. $F_{j k}(y)=1-\bar{F}_{j k}(y)$, Laplace transform $\tilde{f}_{j k}(s)=\int_{0}^{\infty} e^{-s y} f_{j k}(y) d y$ and mean $\mu_{j k}$, if the $i$-th system change involves a claim with $\left(Z_{i-1}, Z_{i}\right)=(j, k)$. Conditional on $\left\{Z_{i}\right\}_{i=0}^{\infty}$, the r.v.'s $X_{1}, X_{2}, \ldots$ are mutually independent as well as being independent of the r.v.'s $W_{1}, W_{2}, \ldots$. Putting together all the above assumptions, it follows that

$$
\operatorname{Pr}\left(X_{i} \leq x, W_{i} \leq y, Z_{i}=k \mid Z_{i-1}=j\right)=K_{j}(y)\left(p_{j k}+q_{j k} F_{j k}(x)\right),
$$

for $x, y \geq 0$.

The surplus process $\underline{U}=\{U(t), t \geq 0\}$ is defined as

$$
U(t)=u+c t-\sum_{i=1}^{N(t)} X_{i}
$$

where $u(u \geq 0)$ is the initial surplus level and $c(c>0)$ is the level premium rate. Let $\tau=$ $\inf \{t \geq 0: U(t)<0\}$ be the time to ruin for the surplus process $\underline{U}$ with $\tau=\infty$ if ruin does not occur (i.e. $U(t) \geq 0$ for all $t \geq 0$ ). We define the Gerber-Shiu discounted penalty function $\phi_{i}(u)$ as (see Gerber and Shiu (1998))

$$
\phi_{i}(u)=E\left[e^{-\delta \tau} w\left(U\left(\tau^{-}\right),|U(\tau)|\right) I(\tau<\infty) \mid U(0)=u, Z_{0}=i\right],
$$

where $\delta \geq 0, w: \mathbb{R}^{+} \times \mathbb{R}^{+} \rightarrow \mathbb{R}$ is the so-called penalty function satisfying some mild integrable conditions and $I(A)$ is the indicator function of the event $A$. However, finding an explicit expression for $\phi_{i}(u)$ in the surplus process $\underline{U}$ seems rather difficult given that the c.d.f. $K_{j}$ has been left unspecified (see Willmot (2007) and Landriault and Willmot (2008) for similar observations in the Sparre Andersen risk model with general interclaim times). Hence, we also consider a special case of the Gerber-Shiu function $\phi_{i}(u)$, namely

$$
v_{i}(u, s)=E\left[e^{-\delta \tau} e^{-s U\left(\tau^{-}\right)} w_{1}(|U(\tau)|) I(\tau<\infty) \mid U(0)=u, Z_{0}=i\right],
$$

by letting $w(x, y)=e^{-s x} w_{1}(y)$ for $s \geq 0$ in (1). In this paper, we develop a methodology to evaluate the Gerber-Shiu function $v_{i}(u, s)$ in the framework of the surplus process $\underline{U}$ under the assumption that the densities $f_{j k}$ have a combination of exponentials representation. More general claim size distributions could have been considered (see Section 5) but the combination of exponential distributions have been preferred due to the simplicity of the ensuing presentation. We also point out that we have chosen to emphasize the dependence of $v_{i}(u, s)$ on $s$, given that $v_{i}(u, s)$ has a slightly different form depending on whether $s=0$ or $s>0$. For the reminder of the paper, we assume that the positive security loading condition

$$
c \sum_{i=1}^{m} \pi_{i} \kappa_{i}>\sum_{i=1}^{m} \pi_{i} \sum_{j=1}^{m} q_{i j} \mu_{i j}
$$

is fulfilled whenever $\delta=0$.

The paper is structured as follows: in Section 2, we rely on the use of a probabilistic approach to identify the form of the solution for $v_{i}(u, s)$ under our distributional assumption on the claim sizes. 
In Section 3, an analytic-based approach is then used to complete the identification of the GerberShiu function $v_{i}(u, s)$. In particular, a system of linear equations is derived whose solution leads to the evaluation of $v_{i}(u, s)$ for $u, s \geq 0$. In Section 4 , an application of the suggested methodology is considered via a numerical example. Finally, some concluding remarks are made in Section 5.

\section{Probabilistic approach}

Let $N_{\tau}$ be the number of system changes up to (and including) ruin and define $h_{i j k}(\delta, x, y)$ as

$$
h_{i j k}(\delta, x, y) d x d y=E\left[\begin{array}{c}
e^{-\delta \tau} I\left(U\left(\tau^{-}\right) \in(x, x+d x)\right) I(|U(\tau)| \in(y, y+d y)) \\
I\left(Z_{N_{\tau}-1}=j\right) I\left(Z_{N_{\tau}}=k\right)
\end{array} \mid U(0)=0, Z_{0}=i\right] .
$$

Note that $h_{i j k}(\delta, x, y)$ is a generalization of the discounted joint density of the surplus prior to ruin $(x)$ and the deficit at ruin (y) given an initial surplus of 0 (see e.g. Gerber and Shiu (1997) and Ren (2007)). As expected, this quantity will play a crucial role in the analysis of the expected discounted penalty function.

To analyze the Gerber-Shiu function $\phi_{i}(u)$, we condition on the type of first drop of the surplus process $\underline{U}$ below its initial level $u$ while keeping track of the underlying states of the environmental process $\underline{J}$ just before and after the drop. We arrive at

$$
\begin{aligned}
\phi_{i}(u) & =\sum_{j=1}^{m} \sum_{k=1}^{m} \int_{0}^{u}\left\{\int_{0}^{\infty} \phi_{k}(u-y) h_{i j k}(\delta, x, y) d x\right\} d y \\
& +\sum_{j=1}^{m} \sum_{k=1}^{m} \int_{u}^{\infty}\left\{\int_{0}^{\infty} w(x+u, y-u) h_{i j k}(\delta, x, y) d x\right\} d y
\end{aligned}
$$

for $i=1,2, \ldots, m$. Note that $h_{i j k}(\delta, x, y)$ can be decomposed as

$$
h_{i j k}(\delta, x, y)=h_{i j}(\delta, x) \frac{q_{j k} f_{j k}(x+y)}{\sum_{l=1}^{m} q_{j l} \bar{F}_{j l}(x)},
$$

where

$$
h_{i j}(\delta, x) d x=E\left[e^{-\delta \tau} I\left(U\left(\tau^{-}\right) \in(x, x+d x)\right) I\left(Z_{N_{\tau}-1}=j\right) \mid U(0)=0, Z_{0}=i\right] .
$$

Equation (5) can be explained as follows:

(a) the surplus prior to ruin is $x$ and the environmental state $Z_{N_{\tau}-1}$ is $j$ with discounted density $h_{i j}(\delta, x)$;

(b) given a surplus prior to ruin of $x$ and $Z_{N_{\tau}-1}=j$, the Markov chain $\left\{Z_{i}\right\}_{i=0}^{\infty}$ shall transit from state $j$ to state $k$ and be accompanied by a claim of size $x+y$ for the deficit at ruin to be $y$ and $Z_{N_{\tau}}=k$. This leads to the term $q_{j k} f_{j k}(x+y)$ in (5). However, given that ruin is assumed to occur in $(\mathrm{a}), q_{j k} f_{j k}(x+y)$ shall be divided by the probability that a claim of size greater than $x$ occurs (causing ruin) whenever the Markov chain $\left\{Z_{i}\right\}_{i=0}^{\infty}$ originates from state $j$ at the time of the claim. Considering all the possible transitions from state $j$ to state $l(l=1,2, \ldots, m)$, this probability is easily found to be $\sum_{l=1}^{m} q_{j l} \bar{F}_{j l}(x)$. 
For future reference, it is helpful to write (5) as

$$
h_{i j k}(\delta, x, y)=h_{i j}(\delta, x) q_{j k}^{*}(x) \frac{f_{j k}(x+y)}{\bar{F}_{j k}(x)},
$$

where

$$
q_{j k}^{*}(x)=\frac{q_{j k} \bar{F}_{j k}(x)}{\sum_{l=1}^{m} q_{j l} \bar{F}_{j l}(x)}
$$

is the probability that the state at ruin is $k$ given that the surplus prior to ruin is $x$ and the state prior to ruin is $j$.

By substituting (6) into (4), one further obtains

$$
\begin{aligned}
\phi_{i}(u) & =\sum_{j=1}^{m} \sum_{k=1}^{m} \int_{0}^{u} \phi_{k}(u-y)\left\{\int_{0}^{\infty} h_{i j}(\delta, x) q_{j k}^{*}(x) \frac{f_{j k}(x+y)}{\bar{F}_{j k}(x)} d x\right\} d y \\
& +\sum_{j=1}^{m} \sum_{k=1}^{m} \int_{u}^{\infty}\left\{\int_{0}^{\infty} w(x+u, y-u) h_{i j}(\delta, x) q_{j k}^{*}(x) \frac{f_{j k}(x+y)}{\bar{F}_{j k}(x)} d x\right\} d y .
\end{aligned}
$$

In particular, for the Gerber-Shiu function $v_{i}(u, s),(7)$ reduces to

$$
\begin{aligned}
v_{i}(u, s) & =\sum_{j=1}^{m} \sum_{k=1}^{m} \int_{0}^{u} v_{k}(u-y, s)\left\{\int_{0}^{\infty} h_{i j}(\delta, x) q_{j k}^{*}(x) \frac{f_{j k}(x+y)}{\bar{F}_{j k}(x)} d x\right\} d y \\
& +\sum_{j=1}^{m} \sum_{k=1}^{m} \int_{u}^{\infty} w_{1}(y-u)\left\{\int_{0}^{\infty} e^{-s(x+u)} h_{i j}(\delta, x) q_{j k}^{*}(x) \frac{f_{j k}(x+y)}{\bar{F}_{j k}(x)} d x\right\} d y .
\end{aligned}
$$

In what follows, we assume that the claim size densities $f_{j k}$ admit the factorization

$$
f_{j k}(x+y)=\sum_{l=1}^{n_{j k}} \eta_{j k l}(x) \varphi_{j k l}(y), \quad x, y>0,
$$

for some functions $\eta_{j k l}(x)$ and $\varphi_{j k l}(y)$ (see Willmot (2007)). As a result, the density (in $y$ ) associated to the residual lifetime distribution of $f_{j k}$ can be expressed as

$$
\frac{f_{j k}(x+y)}{\bar{F}_{j k}(x)}=\sum_{l=1}^{n_{j k}} \eta_{j k l}^{*}(x) \varphi_{j k l}(y),
$$

where $\eta_{j k l}^{*}(x)=\eta_{j k l}(x) /\left\{\sum_{l=1}^{n_{j k}} \eta_{j k l}(x) \int_{0}^{\infty} \varphi_{j k l}(y) d y\right\}$.

Using (10), (8) becomes

$$
\begin{aligned}
v_{i}(u, s) & =\sum_{j=1}^{m} \sum_{k=1}^{m} \sum_{l=1}^{n_{j k}}\left\{\int_{0}^{u} v_{k}(u-y, s) \varphi_{j k l}(y) d y\right\}\left\{\int_{0}^{\infty} h_{i j}(\delta, x) q_{j k}^{*}(x) \eta_{j k l}^{*}(x) d x\right\} \\
& +\sum_{j=1}^{m} \sum_{k=1}^{m} \sum_{l=1}^{n_{j k}}\left\{\int_{u}^{\infty} w_{1}(y-u) \varphi_{j k l}(y) d y\right\}\left\{\int_{0}^{\infty} e^{-s(x+u)} h_{i j}(\delta, x) q_{j k}^{*}(x) \eta_{j k l}^{*}(x) d x\right\} .
\end{aligned}
$$


Letting

$$
\chi_{i j k l}(\delta, s)=\int_{0}^{\infty} e^{-s x} h_{i j}(\delta, x) q_{j k}^{*}(x) \eta_{j k l}^{*}(x) d x
$$

(11) can be reduced to

$$
\begin{aligned}
v_{i}(u, s) & =\sum_{j=1}^{m} \sum_{k=1}^{m} \sum_{l=1}^{n_{j k}} \chi_{i j k l}(\delta, 0)\left\{\int_{0}^{u} v_{k}(u-y, s) \varphi_{j k l}(y) d y\right\} \\
& +\sum_{j=1}^{m} \sum_{k=1}^{m} \sum_{l=1}^{n_{j k}} \chi_{i j k l}(\delta, s)\left\{\int_{0}^{\infty} w_{1}(y) \varphi_{j k l}(u+y) d y\right\} e^{-s u} .
\end{aligned}
$$

Owing to the form of (13), a natural way to analyze $v_{i}(u, s)$ is via Laplace transforms (see Willmot (2007) and Landriault and Willmot (2008)). With that in mind, we further assume that the claim size densities $f_{j k}(j, k=1,2, \ldots, m)$ are distributed as combination of exponentials of the form

$$
f_{j k}(y)=\sum_{l=1}^{n_{j k}} A_{j k l}\left(\beta_{j k l} e^{-\beta_{j k l} y}\right), \quad y>0,
$$

where $\sum_{l=1}^{n_{j k}} A_{j k l}=1$ and $\beta_{j k l}>0$ for $l=1,2, \ldots, n_{j k}$. We remark that some of the $A_{j k l}$ 's are allowed to be negative, as long as (14) is a proper density. It is easy to see that (14) satisfies (9) with $\eta_{j k l}(x)=$ $A_{j k l} e^{-\beta_{j k l} x}$ and $\varphi_{j k l}(y)=\beta_{j k l} e^{-\beta_{j k l} y}$. Therefore, the associated residual lifetime distribution of $f_{j k}$ admits the factorization

$$
\frac{f_{j k}(x+y)}{\bar{F}_{j k}(x)}=\sum_{l=1}^{n_{j k}} \eta_{j k l}^{*}(x)\left(\beta_{j k l} e^{-\beta_{j k l} y}\right),
$$

where $\eta_{j k l}^{*}(x)=A_{j k l} e^{-\beta_{j k l} x} / \sum_{z=1}^{n_{j k}} A_{j k z} e^{-\beta_{j k z} x}$. Clearly, $\sum_{l=1}^{n_{j k}} \eta_{j k l}^{*}(x)=1$ which implies that the residual lifetime density (15) is a different combination of the same exponential densities. Apart from the fact that the class of combinations of exponentials is mathematically tractable, it is also known that the class of combinations of exponentials is dense in the set of continuous distributions defined on $\mathbb{R}^{+}$. See e.g. Dufresne (2007) for the fitting of this class of distributions. A remark will be made in Section 5 regarding the use of more general claim size density than (14).

By defining the Laplace transform $\widetilde{w}_{1}(r)=\int_{0}^{\infty} e^{-r y} w_{1}(y) d y$, taking the Laplace transform on both sides of (13) leads to

$$
\widetilde{v}_{i}(r, s)=\sum_{j=1}^{m} \sum_{k=1}^{m} \sum_{l=1}^{n_{j k}} \chi_{i j k l}(\delta, 0) \widetilde{v}_{k}(r, s) \frac{\beta_{j k l}}{\beta_{j k l}+r}+\xi_{i}(\delta, r, s),
$$

for $i=1,2, \ldots, m$ where

$$
\widetilde{v}_{i}(r, s)=\int_{0}^{\infty} e^{-r u} v_{i}(u, s) d u,
$$

and

$$
\xi_{i}(\delta, r, s)=\sum_{j=1}^{m} \sum_{k=1}^{m} \sum_{l=1}^{n_{j k}} \chi_{i j k l}(\delta, s) \frac{\beta_{j k l}}{s+\beta_{j k l}+r} \widetilde{w}_{1}\left(\beta_{j k l}\right) .
$$

In matrix form, (16) can be rewritten as

$$
\widetilde{\mathbf{v}}(r, s)=\boldsymbol{\Upsilon}(r) \widetilde{\mathbf{v}}(r, s)+\boldsymbol{\Xi}(r, s),
$$


or equivalently as

$$
(\mathbf{I}-\mathbf{\Upsilon}(r)) \widetilde{\mathbf{v}}(r, s)=\boldsymbol{\Xi}(r, s),
$$

where $\widetilde{\mathbf{v}}(r, s)=\left(\widetilde{v}_{1}(r, s), \ldots, \widetilde{v}_{m}(r, s)\right)^{T}, \boldsymbol{\Xi}(r, s)=\left(\xi_{1}(\delta, r, s), \ldots, \xi_{m}(\delta, r, s)\right)^{T}, \mathbf{\Upsilon}(r)$ is an $m \times m$ square matrix with $(i, k)$-th element

$$
[\mathbf{\Upsilon}(r)]_{i k}=\sum_{j=1}^{m} \sum_{l=1}^{n_{j k}} \chi_{i j k l}(\delta, 0) \frac{\beta_{j k l}}{\beta_{j k l}+r}
$$

and $\mathbf{I}$ is an $m \times m$ identity matrix. Assuming that $\mathbf{I}-\mathbf{\Upsilon}(r)$ is invertible, we also have that

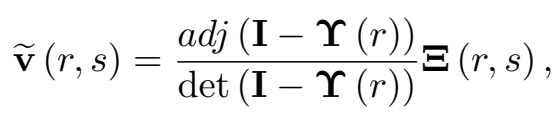

where $\operatorname{adj}(\mathbf{I}-\mathbf{\Upsilon}(r))$ is the adjoint matrix of $\mathbf{I}-\mathbf{\Upsilon}(r)$. Letting

$$
H(r)=\prod_{j=1}^{m} \prod_{k=1}^{m} \prod_{l=1}^{n_{j k}}\left(\beta_{j k l}+r\right),
$$

an equivalent representation for $\widetilde{\mathbf{v}}(r, s)$ is

$$
\widetilde{\mathbf{v}}(r, s)=\frac{H(r) \operatorname{adj}(\mathbf{I}-\mathbf{\Upsilon}(r))}{H(r) \operatorname{det}(\mathbf{I}-\mathbf{\Upsilon}(r))} \mathbf{\Xi}(r, s) .
$$

From the definition of $\mathbf{\Upsilon}(r)$, it is immediate that $H(r) \operatorname{det}(\mathbf{I}-\mathbf{\Upsilon}(r))$ is a polynomial in $r$ of degree $n=\sum_{j=1}^{m} \sum_{k=1}^{m} n_{j k}$ with a leading coefficient of 1 . Let $\left\{\rho_{k}\right\}_{k=1}^{n}$ be the $n$ zeros of $H(r) \operatorname{det}(\mathbf{I}-\mathbf{\Upsilon}(r))$. In what follows, we use a matrix extension of Rouche's theorem (see Theorem 11.3 of de Smit (1995)) to show that the $n$ zeros $\left\{\rho_{k}\right\}_{k=1}^{n}$ have a negative real part. To do so, let $C_{R}$ be the closed contour consisting of the imaginary line running from $-i R$ to $i R$ and the part of the circle of radius $R$ running counterclockwise from $i R$ to $-i R$.

Theorem 1 The $n$ zeros $\left\{\rho_{k}\right\}_{k=1}^{n}$ of $H(r) \operatorname{det}(\mathbf{I}-\mathbf{\Upsilon}(r))$ are such that $\operatorname{Re}\left(\rho_{k}\right)<0$ for $k=1,2, \ldots, n$.

Proof: Let $\mathbf{L}(r)$ be a $m \times m$ diagonal matrix with $[\mathbf{L}(r)]_{i i}=\prod_{j=1}^{m} \prod_{l=1}^{n_{j i}}\left(\beta_{j i l}+r\right)$ for $i=1,2, \ldots, m$. Also, define an $m \times m$ square matrix $\mathbf{B}(r)$ with

$$
[\mathbf{B}(r)]_{i k}=[\mathbf{L}(r)]_{i i}[\boldsymbol{\Upsilon}(r)]_{i k},
$$

for $i, k=1,2, \ldots, m$.

On the semi-circle of $C_{R}$ for $R$ sufficiently large, one has

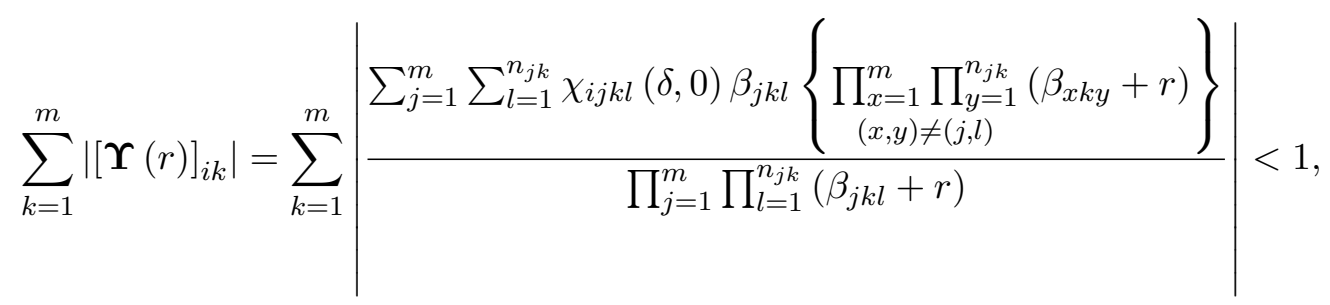


for $i=1,2, \ldots, m$. On the imaginary axis of $C_{R}$, we first substitute (12) at $s=0$ in (17) which yields

$$
[\Upsilon \boldsymbol{\Upsilon}(r)]_{i k}=\sum_{j=1}^{m} \int_{0}^{\infty} h_{i j}(\delta, x) q_{j k}^{*}(x)\left\{\sum_{l=1}^{n_{j k}} \eta_{j k l}^{*}(x) \frac{\beta_{j k l}}{\beta_{j k l}+r}\right\} d x,
$$

where $\sum_{l=1}^{n_{j k}} \eta_{j k l}^{*}(x) \beta_{j k l} /\left(\beta_{j k l}+r\right)$ corresponds to the Laplace transform of the residual lifetime density (15). From (20), it is immediate that

$$
\left|[\Upsilon \Upsilon \Upsilon(r)]_{i k}\right| \leq \sum_{j=1}^{m} \int_{0}^{\infty} h_{i j}(\delta, x) q_{j k}^{*}(x) d x
$$

on the imaginary axis of $C_{R}$. One concludes that

$$
\sum_{k=1}^{m}\left|[\Upsilon \Upsilon(r)]_{i k}\right| \leq \varsigma_{i}(\delta),
$$

where

$$
\varsigma_{i}(\delta)=\sum_{j=1}^{m} \sum_{k=1}^{m} \int_{0}^{\infty} h_{i j}(\delta, x) q_{j k}^{*}(x) d x
$$

is the Laplace transform of the time to ruin given an initial surplus of 0 and $Z_{0}=i$.

For $\delta>0$, it is clear that $0 \leq \varsigma_{i}(\delta)<1$ which implies

$$
\sum_{k=1}^{m}\left|[\mathbf{\Upsilon}(r)]_{i k}\right|<1, \quad i=1,2, \ldots, m,
$$

on the whole contour $C_{R}$ for $R$ sufficiently large. For $\delta=0$, the fact that the Markov chain $\left\{Z_{i}\right\}_{i=0}^{\infty}$ is irreducible together with the positive security loading condition (3) implies that the infinite-time ruin probabilities $\left\{\varsigma_{i}(0)\right\}_{i=1}^{m}$ with an initial surplus of 0 are all strictly less than 1 . This in turn implies that (21) also holds when $\delta=0$. Thus, from (19), one concludes that

$$
\sum_{k=1}^{m}\left|[\mathbf{B}(r)]_{i k}\right| \leq[\mathbf{L}(r)]_{i i}
$$

on $C_{R}$ with $R$ sufficiently large. From Theorem 11.3 of de Smit (1995), the fact that $\operatorname{det}(\mathbf{L}(r))$ has $n$ zeros with a negative real part implies that $\operatorname{det}(\mathbf{L}(r)-\mathbf{B}(r))=H(r) \operatorname{det}(\mathbf{I}-\mathbf{\Upsilon}(r))$ also has $n$ roots with a negative real part.

Equation (18) can now be rewritten as

$$
\widetilde{\mathbf{v}}(r, s)=\frac{H(r) \operatorname{adj}(\mathbf{I}-\mathbf{\Upsilon}(r)) \boldsymbol{\Xi}(r, s)}{\prod_{k=1}^{n}\left(r-\rho_{k}\right)} .
$$

Denoting the $(k, i)$-th element of the cofactor matrix of $\mathbf{I}-\mathbf{\Upsilon}(r)$ by $\operatorname{cof}(\mathbf{I}-\mathbf{\Upsilon}(r))_{k i}$, it follows that the $i$-th element of $\widetilde{\mathbf{v}}(r, s)$ can be expressed as

$$
\widetilde{v}_{i}(r, s)=\frac{\sum_{j=1}^{m} \sum_{z=1}^{m} \sum_{l=1}^{n_{j z}} \widetilde{w}_{1}\left(\beta_{j z l}\right) \frac{\beta_{j z l}}{s+\beta_{j z l}+r} H(r)\left\{\sum_{k=1}^{m} \operatorname{cof}(\mathbf{I}-\mathbf{\Upsilon}(r))_{k i} \chi_{k j z l}(\delta, s)\right\}}{\prod_{k=1}^{n}\left(r-\rho_{k}\right)},
$$


for $i=1,2, \ldots, m$. For simplicity, let us assume that the $\rho_{k}$ 's and the $-\left(s+\beta_{j z l}\right)$ 's are all distinct for a chosen $s \geq 0$. Clearly, (22) can be expressed via partial fractions as

$$
\widetilde{v}_{i}(r, s)=\sum_{k=1}^{n} \frac{a_{i k}(s)}{r-\rho_{k}}+\sum_{j=1}^{m} \sum_{z=1}^{m} \sum_{l=1}^{n_{j z}} \frac{b_{i j z l}(s)}{s+\beta_{j z l}+r},
$$

for some constants $a_{i k}(s)$ 's and $b_{i k z l}(s)$ 's (independent of $r$ ). Thus, a Laplace transform inversion of (23) yields

$$
v_{i}(u, s)=\sum_{k=1}^{n} a_{i k}(s) e^{\rho_{k} u}+\sum_{j=1}^{m} \sum_{z=1}^{m} \sum_{l=1}^{n_{j z}} b_{i j z l}(s) e^{-\left(s+\beta_{j z l}\right) u},
$$

for $i=1,2, \ldots, m$.

We point out that, for $s \geq 0,(24)$ corresponds to the general form of the solution for $v_{i}(u, s)$. When $s=0$, it is further shown in Appendix that (24) holds with $b_{i j z l}(0)=0$ for all $i, j, z=1,2, \ldots, m$ and $l=1,2, \ldots, n_{j z}$. Hence, for $s=0$, the general solution for $v_{i}(u, s)$ reduces to

$$
v_{i}(u, 0)=\sum_{k=1}^{n} a_{i k}(0) e^{\rho_{k} u}
$$

for $i=1,2, \ldots, m$.

\section{Analytic approach}

In this section, we complete the analysis of $v_{i}(u, s)$ by the identification of the unknown constants in the solution form (24) (or (25)) of $v_{i}(u, s)$. Capitalizing on the analytic approach which consists in conditioning on the time of the first system change $W_{1}$, the size of the accompanying claim $X_{1}$ and the environmental state $Z_{1}$, one finds that

$$
\begin{aligned}
\phi_{i}(u) & =\int_{0}^{\infty} e^{-\delta t} k_{i}(t)\left(\sum_{j=1}^{m} p_{i j} \phi_{j}(u+c t)+\sum_{j=1}^{m} q_{i j} \int_{0}^{u+c t} \phi_{j}(u+c t-y) f_{i j}(y) d y\right) d t \\
& +\int_{0}^{\infty} e^{-\delta t} k_{i}(t)\left(\sum_{j=1}^{m} q_{i j} \int_{u+c t}^{\infty} w(u+c t, y-u-c t) f_{i j}(y) d y\right) d t .
\end{aligned}
$$

For the Gerber-Shiu function $v_{i}(u, s)$, one easily deduces that

$$
\begin{aligned}
v_{i}(u, s) & =\int_{0}^{\infty} e^{-\delta t} k_{i}(t)\left(\sum_{j=1}^{m} p_{i j} v_{j}(u+c t, s)+\sum_{j=1}^{m} q_{i j} \int_{0}^{u+c t} v_{j}(u+c t-y, s) f_{i j}(y) d y\right) d t \\
& +e^{-s u} \int_{0}^{\infty} e^{-(\delta+c s) t} k_{i}(t)\left(\sum_{j=1}^{m} q_{i j} \int_{0}^{\infty} w_{1}(y) f_{i j}(y+u+c t) d y\right) d t
\end{aligned}
$$


Given that the claim size densities are of the form (14), (26) becomes

$$
\begin{aligned}
v_{i}(u, s) & =\int_{0}^{\infty} e^{-\delta t} k_{i}(t)\left(\sum_{j=1}^{m} p_{i j} v_{j}(u+c t, s)+\sum_{j=1}^{m} q_{i j} \int_{0}^{u+c t} v_{j}(u+c t-y, s) \sum_{k=1}^{n_{i j}} A_{i j k} \beta_{i j k} e^{-\beta_{i j k} y} d y\right) d t \\
& +e^{-s u} \int_{0}^{\infty} e^{-(\delta+c s) t} k_{i}(t) \sum_{j=1}^{m} q_{i j} \sum_{k=1}^{n_{i j}} A_{i j k} \beta_{i j k} e^{-\beta_{i j k}(u+c t)}\left(\int_{0}^{\infty} w_{1}(y) e^{-\beta_{i j k} y} d y\right) d t \\
& =\int_{0}^{\infty} e^{-\delta t} k_{i}(t)\left(\sum_{j=1}^{m} p_{i j} v_{j}(u+c t, s)+\sum_{j=1}^{m} q_{i j} \int_{0}^{u+c t} v_{j}(u+c t-y, s) \sum_{k=1}^{n_{i j}} A_{i j k} \beta_{i j k} e^{-\beta_{i j k} y} d y\right) d t \\
& +\sum_{j=1}^{m} \sum_{k=1}^{n_{i j}} q_{i j} A_{i j k} \beta_{i j k} \widetilde{w}_{1}\left(\beta_{i j k}\right) \widetilde{k}_{i}\left(\delta+c\left(s+\beta_{i j k}\right)\right) e^{-\left(s+\beta_{i j k}\right) u},
\end{aligned}
$$

for $i=1,2, \ldots, m$. Substituting (24) into (27) results in

$$
\begin{aligned}
& \sum_{l=1}^{n} a_{i l}(s) e^{\rho_{l} u}+\sum_{x=1}^{m} \sum_{z=1}^{m} \sum_{l=1}^{n_{x z}} b_{i x z l}(s) e^{-\left(s+\beta_{x z l}\right) u} \\
& =\sum_{j=1}^{m} p_{i j} \int_{0}^{\infty} e^{-\delta t} k_{i}(t)\left\{\sum_{l=1}^{n} a_{j l}(s) e^{\rho_{l}(u+c t)}+\sum_{x=1}^{m} \sum_{z=1}^{m} \sum_{l=1}^{n_{x z}} b_{j x z l}(s) e^{-\left(s+\beta_{x z l}\right)(u+c t)}\right\} d t \\
& +\sum_{j=1}^{m} q_{i j} \int_{0}^{\infty} e^{-\delta t} k_{i}(t) \int_{0}^{u+c t}\left\{\sum_{l=1}^{n} a_{j l}(s) e^{\rho_{l}(u+c t-y)}\right\}\left(\sum_{k=1}^{n_{i j}} A_{i j k} \beta_{i j k} e^{-\beta_{i j k} y}\right) d y d t \\
& +\sum_{j=1}^{m} q_{i j} \int_{0}^{\infty} e^{-\delta t} k_{i}(t) \int_{0}^{u+c t}\left\{\sum_{x=1}^{m} \sum_{z=1}^{m} \sum_{l=1}^{n_{x z}} b_{j x z l}(s) e^{-\left(s+\beta_{x z l}\right)(u+c t-y)}\right\}\left(\sum_{k=1}^{n_{i j}} A_{i j k} \beta_{i j k} e^{-\beta_{i j k} y}\right) d y d t \\
& +\sum_{z=1}^{m} \sum_{l=1}^{n_{i z}} q_{i z} A_{i z l} \beta_{i z l} \widetilde{w}_{1}\left(\beta_{i z l}\right) \widetilde{k}_{i}\left(\delta+c\left(s+\beta_{i z l}\right)\right) e^{-\left(s+\beta_{i z l}\right) u} .
\end{aligned}
$$

Simple manipulations of (28) further lead to

$$
\begin{aligned}
& \sum_{l=1}^{n} a_{i l}(s) e^{\rho_{l} u}+\sum_{x=1}^{m} \sum_{z=1}^{m} \sum_{l=1}^{n_{x z}} b_{i x z l}(s) e^{-\left(s+\beta_{x z l}\right) u} \\
& =\sum_{l=1}^{n}\left\{\widetilde{k}_{i}\left(\delta-c \rho_{l}\right) \sum_{j=1}^{m}\left(p_{i j}+q_{i j} \sum_{k=1}^{n_{i j}} \frac{A_{i j k} \beta_{i j k}}{\beta_{i j k}+\rho_{l}}\right) a_{j l}(s)\right\} e^{\rho_{l} u} \\
& +\sum_{x=1}^{m} \sum_{z=1}^{m} \sum_{l=1}^{n_{x z}}\left\{\widetilde{k}_{i}\left(\delta+c\left(s+\beta_{x z l}\right)\right) \sum_{j=1}^{m}\left(p_{i j}+q_{i j} \sum_{k=1}^{n_{i j}} \frac{A_{i j k} \beta_{i j k}}{\beta_{i j k}-\left(s+\beta_{x z l}\right)}\right) b_{j x z l}(s)\right\} e^{-\left(s+\beta_{x z l}\right) u} \\
& -\sum_{j=1}^{m} \sum_{k=1}^{n_{i j}}\left\{q_{i j} A_{i j k} \beta_{i j k} \widetilde{k}_{i}\left(\delta+c \beta_{i j k}\right)\left(\sum_{l=1}^{n} \frac{a_{j l}(s)}{\beta_{i j k}+\rho_{l}}+\sum_{x=1}^{m} \sum_{z=1}^{m} \sum_{l=1}^{n_{x z}} \frac{b_{j x z l}(s)}{\beta_{i j k}-\left(s+\beta_{x z l}\right)}\right)\right\} e^{-\beta_{i j k} u} \\
& +\sum_{z=1}^{m} \sum_{l=1}^{n_{i z}} q_{i z} A_{i z l} \beta_{i z l} \widetilde{w}_{1}\left(\beta_{i z l}\right) \widetilde{k}_{i}\left(\delta+c\left(s+\beta_{i z l}\right)\right) e^{-\left(s+\beta_{i z l}\right) u} .
\end{aligned}
$$


Given that (29) holds for $u \geq 0$, equating the coefficient of $e^{\rho_{l} u}$ on both sides of (29) yields

$$
a_{i l}(s)=\widetilde{k}_{i}\left(\delta-c \rho_{l}\right) \sum_{j=1}^{m}\left(p_{i j}+q_{i j} \sum_{k=1}^{n_{i j}} \frac{A_{i j k} \beta_{i j k}}{\beta_{i j k}+\rho_{l}}\right) a_{j l}(s),
$$

for $i=1,2, \ldots, m$ and $l=1,2, \ldots, n$. For each fixed $l=1,2, \ldots, n,(30)$ forms a system of $m$ homogeneous linear equations in $\left\{a_{j l}(s)\right\}_{j=1}^{m}$. Assume that those $n$ systems of equations (30) have a non-trivial solution, i.e. $\left\{a_{j l}(s)\right\}_{j=1}^{m}$ are not all equal to 0 for any given $l=1,2, \ldots, n$. This implies that

$$
\operatorname{det}\left(\mathbf{I}-\boldsymbol{\Lambda}\left(\rho_{l}\right)\right)=0,
$$

for $l=1,2, \ldots, n$ where the $(i, j)$-th element of the $m \times m$ matrix $\boldsymbol{\Lambda}(r)$ is given by $\widetilde{k}_{i}(\delta-c r)\left(p_{i j}+q_{i j} \sum_{k=1}^{n_{i j}} A_{i j k} \beta_{i j k} /\left(\beta_{i j k}+r\right)\right)$. Similarly as in Theorem 1, one can use Theorem 11.3 of de Smit (1995) to show that $\operatorname{det}(\mathbf{I}-\boldsymbol{\Lambda}(r))$ has exactly $n$ zeros with negative real parts. From Theorem 1 together with (31), those $n$ zeros are $\left\{\rho_{l}\right\}_{l=1}^{n}$.

Remark 2 From Theorem 1 and Equation (31), one concludes that $\left\{\rho_{l}\right\}_{l=1}^{n}$ are the $n$ roots with negative real parts of both $\operatorname{det}(\mathbf{I}-\boldsymbol{\Lambda}(r))$ and $H(r) \operatorname{det}(\mathbf{I}-\mathbf{\Upsilon}(r))$. Given that the matrix $\mathbf{\Upsilon}(r)$ involves the quantity $\chi_{i j k l}(\delta, 0)$ for which an expression has not yet been determined, the roots $\left\{\rho_{l}\right\}_{l=1}^{n}$ should be identified as the $n$ solutions with negative real parts of $\operatorname{det}(\mathbf{I}-\boldsymbol{\Lambda}(r))=0$.

\subsection{Case $s>0$}

Let us first consider the case where $s>0$. By equating the coefficients of $e^{-\left(s+\beta_{x z l}\right) u}$ in (29), one obtains

$$
\begin{aligned}
b_{i x z l}(s) & =\widetilde{k}_{i}\left(\delta+c\left(s+\beta_{x z l}\right)\right) \sum_{j=1}^{m}\left(p_{i j}+q_{i j} \sum_{k=1}^{n_{i j}} \frac{A_{i j k} \beta_{i j k}}{\beta_{i j k}-\left(s+\beta_{x z l}\right)}\right) b_{j x z l}(s) \\
& +I(i=x) q_{x z} A_{x z l} \beta_{x z l} \widetilde{w}_{1}\left(\beta_{x z l}\right) \widetilde{k}_{x}\left(\delta+c\left(s+\beta_{x z l}\right)\right),
\end{aligned}
$$

for $i, x, z=1,2, \ldots, m$ and $l=1,2, \ldots, n_{x z}$. Therefore, for each fixed $x, z=1,2, \ldots, m$ and $l=$ $1,2, \ldots, n_{x z},(32)$ for $i=1,2, \ldots, m$ form a system of $m$ linear equations in $\left\{b_{i x z l}(s)\right\}_{i=1}^{m}$. Note this linear system has coefficient matrix $\mathbf{I}-\boldsymbol{\Lambda}\left(-\left(s+\beta_{x z l}\right)\right)$ which has non-zero determinant under the assumption that $\rho_{i}$ 's and $-\left(s+\beta_{j k l}\right)$ 's are all distinct and given that the $\rho_{i}$ 's are the only solutions to $\operatorname{det}(\mathbf{I}-\boldsymbol{\Lambda}(r))=$ 0 with negative real parts. This implies that the solution $\left\{b_{i x z l}(s)\right\}_{i=1}^{m}$ to the linear system (32) is unique.

Similarly, equating the coefficients of $e^{-\beta_{i j k} u}$ in (29) leads to

$$
\sum_{l=1}^{n} \frac{a_{j l}(s)}{\beta_{i j k}+\rho_{l}}+\sum_{x=1}^{m} \sum_{z=1}^{m} \sum_{l=1}^{n_{x z}} \frac{b_{j x z l}(s)}{\beta_{i j k}-\left(s+\beta_{x z l}\right)}=0,
$$

for $i, j=1,2, \ldots, m$ and $k=1,2, \ldots, n_{i j}$. Interestingly, due to the relationship (23), the above equation can equivalently be expressed as

$$
\widetilde{v}_{j}\left(-\beta_{i j k}, s\right)=0
$$

for $i, j=1,2, \ldots, m$ and $k=1,2, \ldots, n_{i j}$. To solve for the $a_{j l}(s)$ 's, we consider the system of equations (30) and remove 1 of the $m$ equations in $i$ for each fixed $l=1,2, \ldots, n$ (given that the $m$ equations are linearly dependent due to (31)). The resulting $(m-1) n$ equations together with the $n$ equations in (33) form a system of $n m$ equations to solve for the $a_{j l}(s)$ 's. 


\section{$3.2 \quad$ Case $s=0$}

By substituting both $s$ and $b_{i x z l}(s)$ for $i, x, z=1,2, \ldots, m$ and $l=1,2, \ldots, n_{x z}$ by $0,(29)$ becomes

$$
\begin{aligned}
\sum_{l=1}^{n} a_{i l}(0) e^{\rho_{l} u} & =\sum_{l=1}^{n}\left\{\widetilde{k}_{i}\left(\delta-c \rho_{l}\right) \sum_{j=1}^{m}\left(p_{i j}+q_{i j} \sum_{k=1}^{n_{i j}} \frac{A_{i j k} \beta_{i j k}}{\beta_{i j k}+\rho_{l}}\right) a_{j l}(0)\right\} e^{\rho_{l} u} \\
& -\sum_{j=1}^{m} \sum_{k=1}^{n_{i j}}\left\{q_{i j} A_{i j k} \beta_{i j k} \widetilde{k}_{i}\left(\delta+c \beta_{i j k}\right) \sum_{l=1}^{n} \frac{a_{j l}(0)}{\beta_{i j k}+\rho_{l}}\right\} e^{-\beta_{i j k} u} \\
& +\sum_{z=1}^{m} \sum_{l=1}^{n_{i z}} q_{i z} A_{i z l} \beta_{i z l} \widetilde{w}_{1}\left(\beta_{i z l}\right) \widetilde{k}_{i}\left(\delta+c \beta_{i z l}\right) e^{-\beta_{i z l} u} .
\end{aligned}
$$

Equating the coefficients of $e^{\rho_{l} u}$ on both sides of (34) results in (30) for $s=0$. Similarly, equating the coefficients of $e^{-\beta_{i j k} u}$ yields

$$
\sum_{l=1}^{n} \frac{a_{j l}(0)}{\beta_{i j k}+\rho_{l}}=\widetilde{w}_{1}\left(\beta_{i j k}\right)
$$

or equivalently,

$$
\widetilde{v}_{j}\left(-\beta_{i j k}, 0\right)=-\widetilde{w}_{1}\left(\beta_{i j k}\right),
$$

for $i, j=1,2, \ldots, m$ and $k=1,2, \ldots, n_{i j}$. To solve for the $a_{j l}(0)$ 's, we consider the system of equations (30) at $s=0$ and remove 1 of the $m$ equations in $i$ for each fixed $l=1,2, \ldots, n$. The resulting $(m-1) n$ equations together with the $n$ equations in (35) form a system of $n m$ equations to solve for the $a_{j l}(0)$ 's.

\section{A numerical example}

In this section, we rely on a numerical example to highlight two important features. On one hand, we illustrate the tractability of the methodology proposed in Sections 2 and 3 for the study of Gerber-Shiu functions. On the other hand, we show that the choice of the distributions for the waiting time between two successive system changes can have a significant impact on the resulting values of some ruin-related quantities. In this section, we look at both the ruin probability and the expected deficit at ruin.

Let us consider a two-state model with probability matrices of system changes

$$
\mathbf{P}=\left[\begin{array}{cc}
0 & 1 / 10 \\
1 / 5 & 0
\end{array}\right] \quad \text { and } \quad \mathbf{Q}=\left[\begin{array}{cc}
4 / 5 & 1 / 10 \\
2 / 5 & 2 / 5
\end{array}\right]
$$

For the Markov chain $\left\{Z_{i}\right\}_{i=0}^{\infty}$, the long-run proportions of time in state 1 and 2 are found to be $\pi_{1}=3 / 4$ and $\pi_{2}=1 / 4$. Moreover, the claim size densities are assumed to be

$$
\begin{aligned}
& f_{11}(y)=2 e^{-2 y}, \quad f_{12}(y)=\frac{1}{3}\left(\frac{1}{6} e^{-\frac{1}{6} y}\right)+\frac{2}{3}\left(\frac{2}{3} e^{-\frac{2}{3} y}\right) \\
& f_{21}(y)=e^{-y} \quad \text { and } \quad f_{22}(y)=\frac{1}{5}\left(\frac{1}{10} e^{-\frac{1}{10} y}\right)+\frac{4}{5}\left(\frac{1}{5} e^{-\frac{1}{5} y}\right)
\end{aligned}
$$


with means $\mu_{11}=1 / 2, \mu_{12}=3, \mu_{21}=1$ and $\mu_{22}=6$ respectively.

We consider a total of 3 scenarios in which the distributions for the waiting time between two successive system changes vary. We choose the distribution of the waiting times to be of one of three types: exponential, gamma or Pareto (denoted by $\operatorname{Exp}(\beta), \operatorname{Gamma}(\alpha, \beta)$ and $\operatorname{Pareto}(\alpha, \theta)$ ) having densities

$$
k(t)=\beta e^{-\beta t}, \quad k(t)=\frac{\beta^{\alpha} t^{\alpha-1} e^{-\beta t}}{\Gamma(\alpha)} \quad \text { and } \quad k(t)=\frac{\alpha \theta^{\alpha}}{(t+\theta)^{\alpha+1}}
$$

respectively. The distributional assumptions on the $k_{1}$ and $k_{2}$ densities for the three scenarios are summarized in Table 1:

\begin{tabular}{|c|c|c|}
\hline Scenario & Distribution of $k_{1}$ & Distribution of $k_{2}$ \\
\hline 1 & $\operatorname{Exp}(2 / 3)$ & $\operatorname{Exp}(2)$ \\
\hline 2 & Gamma $(3 / 2,1)$ & $\operatorname{Gamma}(5 / 2,5)$ \\
\hline 3 & Pareto $(4,9 / 2)$ & Pareto $(7,3)$ \\
\hline
\end{tabular}

Table 1 : Distributional assumptions on $k_{1}$ and $k_{2}$

Note that the three $k_{1}\left(k_{2}\right)$ densities have mean $\kappa_{1}=3 / 2\left(\kappa_{2}=1 / 2\right)$. Under Scenarios 1-3, the variances of the $k_{1}$ densities are $2.25,1.5$ and 4.5 respectively whereas the variances of the $k_{2}$ densities are easily found to be $0.25,0.1$ and 0.35 . Finally, a premium rate of $c=3 / 2$ is assumed so that the positive security loading condition (3) holds.

Note that one could consider Scenario 1, a MAP risk model, to be the baseline scenario. Scenarios 2 and 3 are generalizations of the MAP risk models with respectively lower and higher variances for the times between successive system changes in both states. We remark that state 1 of the Markov chain $\left\{Z_{i}\right\}_{i=0}^{\infty}$ can be regarded as the 'normal' state while state 2 can be viewed as the 'infectious' state. Claims associated with state 1 have smaller mean than those associated with state 2 . On the other hand, the mean waiting time until a system change in state 1 is larger than that in state 2 , meaning that system changes (and also claims) are less frequent in state 1 than state 2.

Example 3 (Ruin probability) The ruin probability $\operatorname{Pr}\left(\tau<\infty \mid U(0)=u, Z_{0}=i\right)$ of the surplus process $\underline{U}=\{U(t), t \geq 0\}$ is a special case of the Gerber-Shiu function (2) with $\delta=s=0$ and $w_{1} \equiv 1$. In Figure 1 , the ruin probabilities under the three scenarios are plotted against the initial surplus level $u$.

\section{INSERT FIGURE 1}

From the above description of the two states of the Markov chain $\left\{Z_{i}\right\}_{i=0}^{\infty}$, one expects the ruin probability with $Z_{0}=2$ to be greater than the ruin probability with $Z_{0}=1$ for a given scenario and initial surplus level. Such behavior is exhibited in Figure 1. Also, for $Z_{0}=i(i=1,2)$, one observes that the ruin probability increases with the variances of the $k_{1}$ and $k_{2}$ densities. This is consistent with an observation made by Landriault and Willmot (2008). Indeed, recall that the mean of the $k_{1}$ and $k_{2}$ densities are fixed under all three scenarios. A distribution with a large variance (e.g. Pareto distribution) is more likely to have extremes when compared to a distribution with identical mean and lower variance. Consequently, for a heavy-tailed distribution, a system change (and hence a claim) can occur shortly with a larger probability than a lighter tailed distribution. Under this scenario, the occurrence of a claim will likely produce ruin. 
Example 4 (Expected deficit at ruin) The expected deficit at ruin $E\left[|U(\tau)| I(\tau<\infty) \mid U(0)=u, Z_{0}=i\right]$ is also a special of the Gerber-Shiu function (2) with $\delta=s=0$ and $w_{1}(y)=y$. A plot of the expected deficit at ruin against the initial surplus level $u$ under the three scenarios in Table 1 can be found in Figure 2.

INSERT FIGURE 2

Here again, the choice of the waiting time distributions has a significant impact on the values of the expected deficit at ruin. Also, we observe that the ordering of the six lines in Figure 2 (to the exception of small initial surplus levels) is identical to that for the ruin probability in Figure 1.

\section{Concluding remarks}

This paper generalizes the MAP risk model by allowing the waiting times between two successive system changes to have arbitrary distributions. The analysis of risk models with such general waiting times has usually been performed under additional assumptions on the claim size distributions (see Willmot (2007) and Landriault and Willmot (2008)). In this paper, we have chosen claim size densities of the form (14). Along the same lines as Landriault and Willmot (2008), it is clear that the present analysis can be extended to claim size densities of the form

$$
f_{j k}(y)=\sum_{l=1}^{n_{j k}} \sum_{a=1}^{m_{j k l}} A_{j k l a} \frac{\left(\beta_{j k l}\right)^{a} y^{a-1} e^{-\beta_{j k l} y}}{(a-1) !}
$$

for $j, k=1,2, \ldots, m$ where $\sum_{l=1}^{n_{j k}} \sum_{a=1}^{m_{j k l}} A_{j k l a}=1$. It can be verified that the density (36) indeed admits the factorization (9). In this context, the solution (24) to the Gerber-Shiu function $v_{i}(u, s)$ becomes

$$
v_{i}(u, s)=\sum_{k=1}^{n} a_{i k}(s) e^{\rho_{k} u}+\sum_{j=1}^{m} \sum_{z=1}^{m} \sum_{l=1}^{n_{j z}} \sum_{a=1}^{m_{j z l}} b_{i j z l a}(s)\left\{u^{a-1} e^{-\left(s+\beta_{j z l}\right) u}\right\},
$$

with $n=\sum_{j=1}^{m} \sum_{z=1}^{m} \sum_{l=1}^{n_{j z}} m_{j z l}$. However, the form of the solution (37) would result in a more lengthy Section 3 without gaining additional insights into the problem (and possibly distract the reader from it). This is why here the claim size densities (14) have been preferred over the more general densities (36).

We conclude this paper by a brief comment on the possible application of our generalized Markov risk model to the analysis of a generalization to the Markov-modulated fluid flow (MMFF) model in the fluid literature. Fluid models have found applications in a wide variety of areas including network performance, storage and inventory processes, financial and insurance risk models. Several authors (see e.g. Ahn and Ramaswami (2004), Asmussen (1995), Rogers (1994) and references therein) have analyzed stochastic fluid flow models using a variety of methods. Capitalizing on the well-known connection between fluid flows and risk processes which primarily consists of replacing downward jumps (e.g. claim sizes) in the risk process by downward linear paths in the fluid process (see Asmussen (1995) for more details), the risk model introduced in this paper may be interpreted in terms of a stochastic fluid flow model that 
extends the popular MMFF in the fluid literature. As an immediate consequence of this observation, some quantities of interest for the generalized MMFF including the Laplace transform of a busy period can be readily obtained from the present analysis of $v_{i}(u, s)$ in this paper. We also refer the interested readers to Latouche and Tetsuya (2004), who analyzed the structure of the stationary distribution for fluid queues controlled by two subclasses of semi-Markov processes.

Acknowledgements: The authors would like to thank the anonymous referee for comments which improved the paper. Support for Eric C.K. Cheung from a start-up fund provided by the Faculty of Science and the Department of Statistics and Actuarial Science as well as the Seed Funding for Basic Research (Project number: 201103159001) provided by the University Research Committee at the University of Hong Kong is also gratefully acknowledged. Andrei Badescu and David Landriault gratefully acknowledges financial support received from the Natural Sciences and Engineering Research Council of Canada (NSERC).

\section{References}

- Ahn, Soohan and Ramaswami, Vaidyanathan. 2004. Transient analysis of fluid flow models via stochastic coupling to a queue. Stochastic Models 20(1): 71-101.

- Ahn, Soohan, Badescu, Andrei L. and Ramaswami, Vaidyanathan. 2007. Time dependent analysis of finite buffer fluid flows and risk models with a dividend barrier. Queueing Systems 55(4): $207-222$.

- Albrecher, Hansjörg and Boxma, Onno J. 2005. On the discounted penalty function in a Markov-dependent risk model. Insurance: Mathematics and Economics 37(3): 650-672.

- Asmussen, Søren. 1989. Risk theory in a Markovian environment. Scandinavian Actuarial Journal 1989: 69-100.

- Asmussen, Søren. 1995. Stationary distributions for fluid flow models with or without brownian noise. Stochastic Models, 11(1): 21-49.

- Badescu, Andrei l., Breuer, lothar, Da Silva Soares, Ana, Latouche, Guy, Remiche, Marie-Ange And Stanford, David. 2005. Risk processes analyzed as fluid queues. Scandinavian Actuarial Journal 2005(2): 127-141.

- Cheung, Eric C.K., And Landriault, David. 2009. Perturbed MAP risk models with dividend barrier strategies. Journal of Applied Probability 46(2): 521-541.

- De Smit, Jos H.A. 1995. Explicit Wiener-Hopf factorization for the analysis of multi-dimensional queues. In: Dshalalow, Jewgeni H. (Ed.) Advances in Queueing: Theory, Methods and Open Problems., Boca Raton: CRC Press, pp. 293-310.

- Dufresne, Daniel. 2007. Fitting combinations of exponentials to probability distributions. Applied Stochastic Models in Business and Industry 23(1): 23-48.

- Gerber, Hans U. and Shiu, Elias S.W. 1997. The joint distribution of the time of ruin, the surplus immediately before ruin, and the deficit at ruin. Insurance: Mathematics and Economics 21(2): 129-137. 
- Gerber, Hans U. and Shiu, Elias S.W. 1998. On the time value of ruin. North American Actuarial Journal 2(1): 48-78.

- Landriault, David and Willmot, Gordon E. 2008. On the Gerber-Shiu discounted penalty function in the Sparre Andersen model with an arbitrary interclaim time distribution. Insurance: Mathematics and Economics 42(2): 600-608.

- Latouche, Guy and Takine, Tetsuya. 2004. Markov-renewal fluid queues. Journal of Applied Probability 41(3): 746-757.

- Li, Shuanming And Lu, Yi. 2007. Moments of the dividend payments and related problems in a Markov-modulated risk model. North American Actuarial Journal 11(2): 65-76.

- Neuts, Marcel F. 1979. A versatile Markovian point process. Journal of Applied Probability 16(4): 764-779.

- Ren, Jiandong. 2007. The discounted joint probability of the surplus prior to ruin and the deficit at ruin. North American Actuarial Journal 11(3): 128-136.

- Rogers, L.C.G. 1994. Fluid flow models in queueing theory and Wiener-Hopf factorization of Markov chains. Annals of Applied Probability 4(2): 390-413.

- Willmot, Gordon E. 2007. On the discounted penalty function in the renewal risk model with general interclaim times. Insurance: Mathematics and Economics 41(1): 17-31. 


\section{Appendix}

In this appendix, we show that the general solution $(24)$ for $v_{i}(u, s)$ can be reduced to $(25)$ when $s=0$.

From $(22)$ at $s=0$, we know that the Laplace transform $\widetilde{v}_{i}(r, 0)$ satisfies

$$
\widetilde{v}_{i}(r, 0)=\frac{\sum_{j=1}^{m} \sum_{z=1}^{m} \sum_{l=1}^{n_{j z}} \widetilde{w}_{1}\left(\beta_{j z l}\right) \frac{\beta_{j z l}}{\beta_{j z l}+r} P_{i j z l}(r)}{\prod_{k=1}^{n}\left(r-\rho_{k}\right)}
$$

for $i=1,2, \ldots, m$ where

$$
P_{i j z l}(r)=H(r)\left\{\sum_{k=1}^{m} \operatorname{cof}(\mathbf{I}-\mathbf{\Upsilon}(r))_{k i} \chi_{k j z l}(\delta, 0)\right\}
$$

is a polynomial of degree $n$ in $r$. In what follows, we show that the polynomial $P_{i j z l}(r)$ has a zero at $r=-\beta_{j z l}$ for any $i, j, z=1,2, \ldots, m$ and $l=1,2, \ldots, n_{j z}$.

First, we consider the case $z=i$. Letting

$$
H_{-i}(r)=\frac{H(r)}{\prod_{j=1}^{m} \prod_{l=1}^{n_{j i}}\left(\beta_{j i l}+r\right)}=\prod_{j=1}^{m} \prod_{\substack{k=1 \\ k \neq i}}^{m} \prod_{l=1}^{n_{j k}}\left(\beta_{j k l}+r\right)
$$

it follows that

$$
P_{i j z l}(r)=\left(\prod_{j=1}^{m} \prod_{l=1}^{n_{j i}}\left(\beta_{j i l}+r\right)\right) H_{-i}(r)\left\{\sum_{k=1}^{m} \operatorname{cof}(\mathbf{I}-\Upsilon \mathbf{\Upsilon}(r))_{k i} \chi_{k j i l}(\delta, 0)\right\},
$$

where $H_{-i}(r)\left\{\sum_{k=1}^{m} \operatorname{cof}(\mathbf{I}-\mathbf{\Upsilon}(r))_{k i} \chi_{k j i l}(\delta, 0)\right\}$ is a polynomial of degree $n-\sum_{j=1}^{m} n_{j i}$ in $r$. Hence, it is immediate from the representation (40) that $P_{i j i l}(r)$ has a zero at $r=-\beta_{j i l}$ for $i, j=1,2, \ldots, m$ and $l=1,2, \ldots, n_{j i}$.

Next, we consider the case $z<i$. Define $\mathbf{A}_{k, i}(r)$ as the $(m-1) \times(m-1)$ matrix that results from deleting the $k$-th row and the $i$-th column of $\mathbf{I}-\mathbf{\Upsilon}(r)$. Also, let $\mathbf{A}_{k j, i z}(r)$ be the matrix resulting from taking out rows $k$ and $j$, as well as columns $i$ and $z$ of $\mathbf{I}-\mathbf{\Upsilon}(r)$. Given that $\operatorname{cof}(\mathbf{I}-\mathbf{\Upsilon}(r))_{k i}=$ $(-1)^{k+i} \operatorname{det}\left(\mathbf{A}_{k, i}(r)\right)$, the Laplace expansion of $\operatorname{det}\left(\mathbf{A}_{k, i}(r)\right)$ along the $z$-th column of $\mathbf{A}_{k, i}(r)$ yields

$$
\operatorname{cof}(\mathbf{I}-\mathbf{\Upsilon}(r))_{k i}=(-1)^{k+i}\left\{\begin{array}{c}
\sum_{x=1}^{k-1}\left(I(x=z)-[\mathbf{\Upsilon}(r)]_{x z}\right)(-1)^{z+x} \operatorname{det}\left(\mathbf{A}_{k x, i z}(r)\right) \\
+\sum_{x=k+1}^{m}\left(I(x=z)-[\mathbf{\Upsilon}(r)]_{x z}\right)(-1)^{z+x-1} \operatorname{det}\left(\mathbf{A}_{k x, i z}(r)\right)
\end{array}\right\} .
$$


Using (41) and (17), it follows from (39) that

$$
\begin{aligned}
& P_{i j z l}(r) \\
& =H(r) \sum_{k=1}^{m}(-1)^{k+i} \chi_{k j z l}(\delta, 0)\left\{\begin{array}{r}
\sum_{x=1}^{k-1}\left(I(x=z)-[\mathbf{\Upsilon}(r)]_{x z}\right)(-1)^{z+x} \operatorname{det}\left(\mathbf{A}_{k x, i z}(r)\right) \\
+\sum_{x=k+1}^{m}\left(I(x=z)-[\mathbf{\Upsilon}(r)]_{x z}\right)(-1)^{z+x-1} \operatorname{det}\left(\mathbf{A}_{k x, i z}(r)\right)
\end{array}\right\} \\
& =H(r) \sum_{k=1}^{m}(-1)^{k+i+z+x} \chi_{k j z l}(\delta, 0)\left\{\begin{array}{l}
\sum_{x=1}^{k-1}\left(I(x=z)-\sum_{a=1}^{m} \sum_{b=1}^{n_{a z}} \chi_{x a z b}(\delta, 0) \frac{\beta_{a z b}}{\beta_{a z b}+r}\right) \operatorname{det}\left(\mathbf{A}_{k x, i z}(r)\right) \\
-\sum_{x=k+1}^{m}\left(I(x=z)-\sum_{a=1}^{m} \sum_{b=1}^{n_{a z}} \chi_{x a z b}(\delta, 0) \frac{\beta_{a z b}}{\beta_{a z b}+r}\right) \operatorname{det}\left(\mathbf{A}_{k x, i z}(r)\right)
\end{array}\right\} .
\end{aligned}
$$

Considering the term $\beta_{j z l} /\left(\beta_{j z l}+r\right)$ separately in (42), one finds that

$$
\begin{aligned}
& P_{i j z l}(r) \\
& =H(r) \sum_{k=1}^{m}(-1)^{k+i+z+x} \chi_{k j z l}(\delta, 0)\left\{\begin{array}{c}
\sum_{x=1}^{k-1}\left(I(x=z)-\sum_{\substack{a=1 \\
(a, b) \neq(j, l)}}^{m} \sum_{\substack{n_{a z} \\
\chi_{x a z b}}}(\delta, 0) \frac{\beta_{a z b}}{\beta_{a z b}+r}\right) \operatorname{det}\left(\mathbf{A}_{k x, i z}(r)\right) \\
-\sum_{\substack{x=k+1 \\
m}}^{m}\left(I(x=z)-\sum_{\substack{a=1 \\
(a, b) \neq(j, l)}}^{n_{a z}} \chi_{x a z b}(\delta, 0) \frac{\beta_{a z b}}{\beta_{a z b}+r}\right) \operatorname{det}\left(\mathbf{A}_{k x, i z}(r)\right)
\end{array}\right\} \\
& -H(r) \sum_{k=1}^{m}(-1)^{k+i+z+x} \chi_{k j z l}(\delta, 0) \frac{\beta_{j z l}}{\beta_{j z l}+r}\left\{\begin{array}{c}
\sum_{x=1}^{k-1} \chi_{x j z l}(\delta, 0) \operatorname{det}\left(\mathbf{A}_{k x, i z}(r)\right) \\
-\sum_{x=k+1}^{m} \chi_{x j z l}(\delta, 0) \operatorname{det}\left(\mathbf{A}_{k x, i z}(r)\right)
\end{array}\right\} .
\end{aligned}
$$

A closer examination to the term $\operatorname{det}\left(\mathbf{A}_{k x, i z}(r)\right)$ reveals that the first term of the right-hand side of (43) is 0 when $r=-\beta_{j z l}$. For the second term, a change in the order of summation yields

$$
\begin{aligned}
& \sum_{k=1}^{m}(-1)^{k+i+z+x} \chi_{k j z l}(\delta, 0) \frac{\beta_{j z l}}{\beta_{j z l}+r}\left\{\begin{array}{c}
\sum_{x=1}^{k-1} \chi_{x j z l}(\delta, 0) \operatorname{det}\left(\mathbf{A}_{k x, i z}(r)\right) \\
-\sum_{x=k+1}^{m} \chi_{x j z l}(\delta, 0) \operatorname{det}\left(\mathbf{A}_{k x, i z}(r)\right)
\end{array}\right\} \\
& =\frac{\beta_{j z l}}{\beta_{j z l}+r} \sum_{k=1}^{m} \sum_{x=1}^{k-1}(-1)^{k+x+z+i} \chi_{k j z l}(\delta, 0) \chi_{x j z l}(\delta, 0) \operatorname{det}\left(\mathbf{A}_{k x, i z}(r)\right) \\
& -\frac{\beta_{j z l}}{\beta_{j z l}+r} \sum_{k=1}^{m} \sum_{x=k+1}^{m}(-1)^{k+z+x+i} \chi_{k j z l}(\delta, 0) \chi_{x j z l}(\delta, 0) \operatorname{det}\left(\mathbf{A}_{k x, i z}(r)\right) \\
& =\frac{\beta_{j z l}}{\beta_{j z l}+r} \sum_{x=1}^{m} \sum_{k=x+1}^{m}(-1)^{k+x+z+i} \chi_{k j z l}(\delta, 0) \chi_{x j z l}(\delta, 0) \operatorname{det}\left(\mathbf{A}_{k x, i z}(r)\right) \\
& -\frac{\beta_{j z l}}{\beta_{j z l}+r} \sum_{k=1}^{m} \sum_{x=k+1}^{m}(-1)^{k+z+x+i} \chi_{k j z l}(\delta, 0) \chi_{x j z l}(\delta, 0) \operatorname{det}\left(\mathbf{A}_{k x, i z}(r)\right) \\
& =0 .
\end{aligned}
$$


One concludes that for $z<i$ the polynomial $P_{i j z l}(r)$ has a zero at $r=-\beta_{j z l}$. The proof for $z>i$ follows along the same lines as the one for $z<i$, and is therefore omitted here.

Finally, given that $P_{i j z l}(r)$ has a zero at $r=-\beta_{j z l}$ for any $i, j, z=1,2, \ldots, m$ and $l=1,2, \ldots, n_{j z}$, the numerator on the right-hand side of (38) is a polynomial of degree at most $n-1$ in $r$. Using partial fractions, the Laplace transform inversion of (38) yields (25). 
Ruin probability

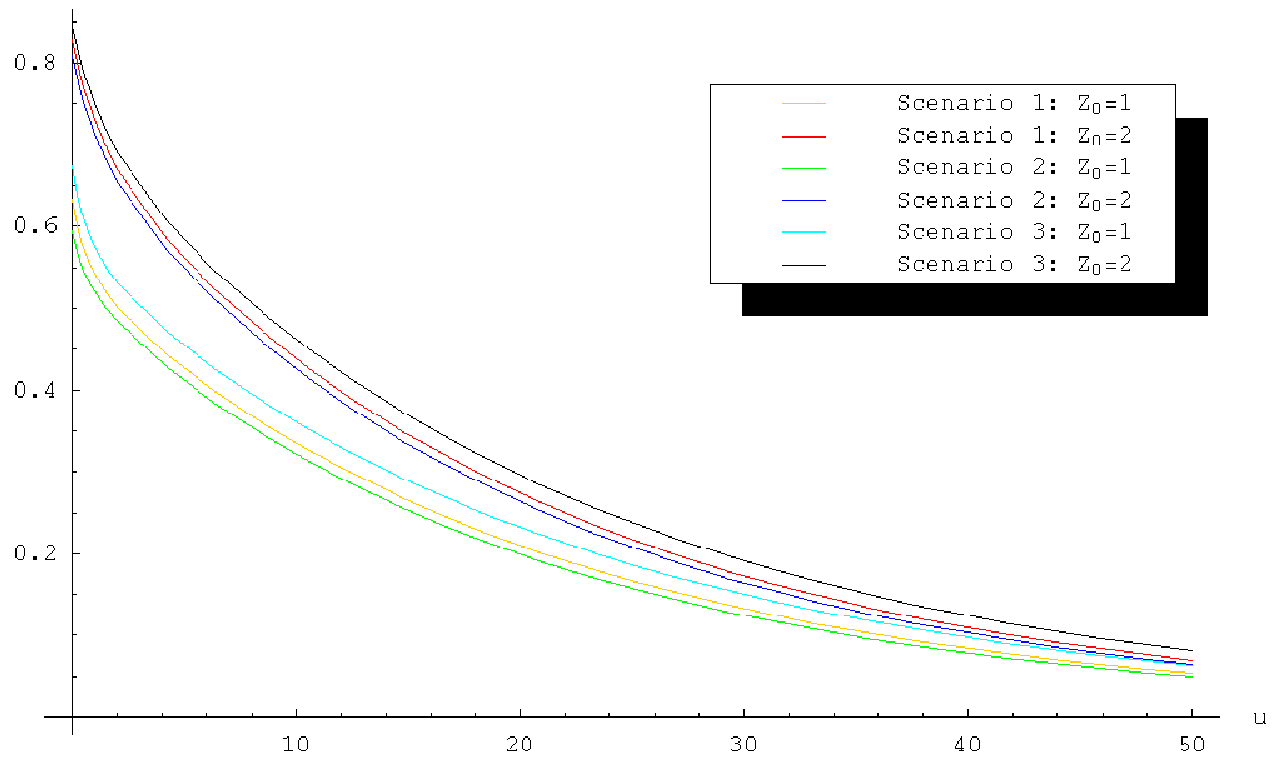

Figure 1

Expected deficit

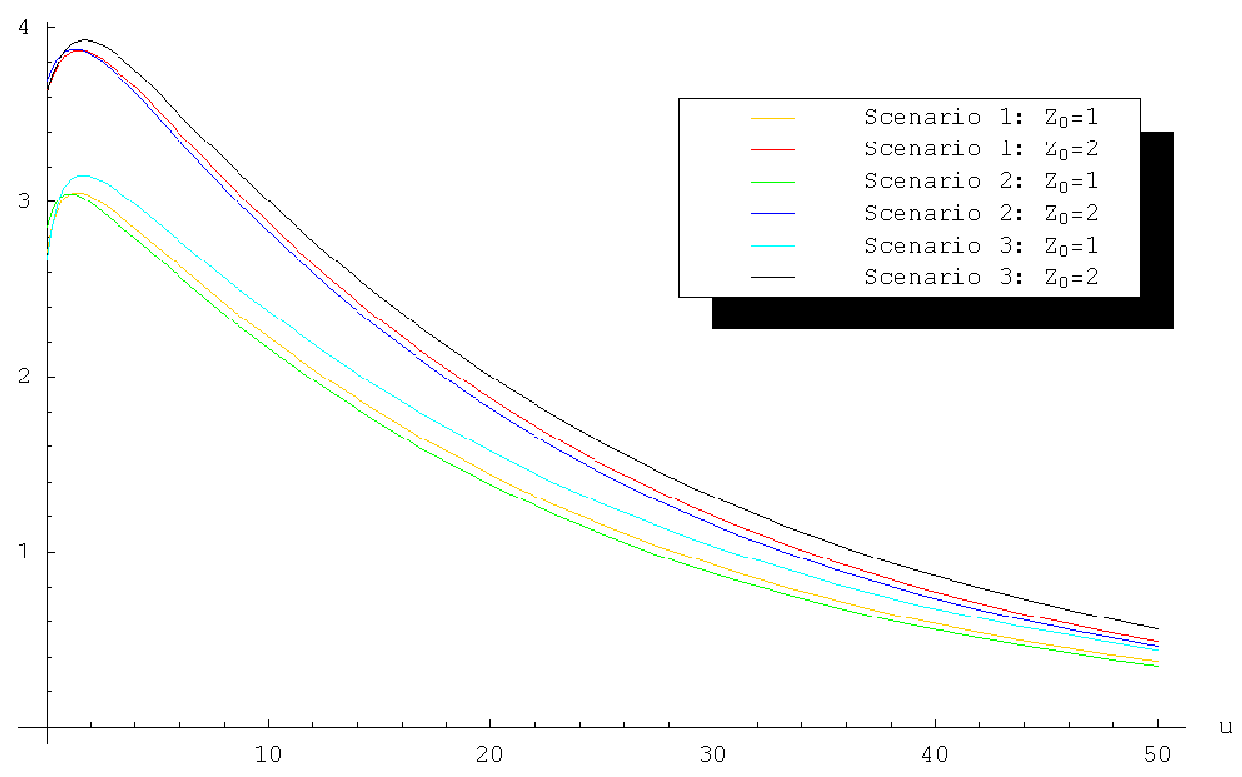

Figure 2 